\title{
A Ghost Story: Ghosts and Gluons in the IR regime of QCD
}

\author{
Olivier Pène* \\ LPT Orsay $(C N R S)^{\dagger}$ \\ E-mail: olivier.pene@th.u-psud.fr
}

Ph. Boucaud

LPT Orsay (CNRS)

E-mail: philippe.boucaudeth.u-psud.fr

\author{
J.P. Leroy \\ LPT Orsay (CNRS) \\ E-mail: jean-pierre.leroy@th.u-psud.fr
}

\section{A. Le Yaouanc \\ LPT Orsay (CNRS) \\ E-mail: alain.le-yaouanceth.u-psud.fr \\ J. Micheli \\ LPT Orsay (CNRS) \\ E-mail: jacques.micheli@th.u-psud.fr}

\section{J. Rodríguez-Quintero}

Dpto. Física Aplicada, Huelva

E-mail: jose.rodriguez@dfaie.uhu.es

We discuss the different methods to obtain reliable informations about the deep infra-red behaviour of the gluon and ghost Green functions in QCD. We argue that a clever combination of analytical inputs and numerical ones is necessary. We illustrate this statement about the distinction between two classes of solutions of the ghost propagator Dyson-Schwinger equation (GPDSE). We conclude that the solution II ("decoupling") with a finite renormalised ghost dressing function at zero momentum is strongly favored by lattice QCD, We derive a method to solve numerically the GPDSE using lattice inputs concerning the gluon propagator. We derive an analytical small momentum expansion of the Ghost dressing function. We prove from the large cut-off behaviour of the ghost propagator renormalisation constant, $\widetilde{Z}_{3}$, that the bare ghost dressing function is infinite at the infinite cut-off limit.

International Workshop on QCD Green's Functions, Confinement and Phenomenology

September 7-11, 2009

ECT Trento, Italy

\footnotetext{
* Speaker.

†Laboratoire de Physique Théorique, Unité Mixte de Recherche 8627 du Centre National de la Recherche Scientifique Université de Paris XI, Bâtiment 210, 91405 Orsay Cedex, France

${ }^{\ddagger}$ Fac. Ciencias Experimentales, Universidad de Huelva, 21071 Huelva, Spain
} 


\section{Introduction}

During the ten years ranging from 1965 to 1974 was invented the standard model of particle physics. This was a major and often overlooked scientific event. During the early sixties, the four fundamental interactions where known, but the weak interaction was only described by Fermi's effective theory and the strong interaction seemed to be even further away from any sound theoretical description, precisely because, being strong, it seemed impossible to control by expanding around a small parameter, as had been the case for quantum electrodynamics.

Then came the miracle. A quantum field theory containing quarks and gauge particles named gluons was proposed and its major property was isolated: asymptotic freedom. It was a miracle because its formulation is extremely compact, with only $n_{f}+1$ free parameters ${ }^{1}$ naming $n_{f}$ the number of quark flavors, i.e. $n_{f}=6$, the beautiful constraints of gauge symmetry, while the field of its applications is huge. Up to now no strong argument has been presented which could allow to deny QCD to be the theory of strong interactions. Of course there are drawbacks. The first is that we are not able to extract very accurate predictions from QCD's premises.

But the most frustrating unsolved problem is the inexistence of a real proof of the confinement property, i.e. of the observation that only hadrons are observed in nature and never isolated quarks or gluons. We are all convinced that confinement is a property of QCD. Confinement is an experimental fact. Furthermore lattice-QCD (LQCD) calculations, which are based on QCD's principles, provide results in full agreement with confinement. But this is not a proof.

Confinement is the major issue of this meeting and we all believe that it has to be looked for in the infrared behaviour of QCD. We will hear in this conference discussions around several approaches to confinement. Our approach will not be to follow or criticise some confinement scenarios, but rather to try to provide reliable answers to the question: How do Green functions behave in the deep infrared. In this talk we will, for the sake of simplicity, restrict ourselves to the quarkless pure Yang-Mills theory. We assume that the main features of QCD's infrared properties are present in Yang-Mills, at odds with Gribov's hypothesis that the light quark supercritical binding was the origin of confinement [1].

\subsection{Tools to handle the Green functions in the deep infrared}

There exist analytical tools which are mainly Ward-Slavnov-Taylor identities (WSTI) and Dyson-Schwinger equations (DSE). There exists a numerical tool which is LQCD.

WSTI and DSE are exact. They can be derived rigorously from the path integral formulation of QCD. However, WSTI's are a necessary a posteriori check but do not constrain so much while DSE's are a very large set of coupled non linear integral equations. Trying to solve the latter is a formidable task and it is not clear how many solutions exist. There can even be an infinity of them.

LQCD is exact, it is really an approximation of QCD, however it is only numerical, leading to an intrinsic uncertainty, and, as we already mentioned, the accuracy is poor.

We believe that it is extremely fruitful to combine both the analytical and numerical approaches. Indeed we will see an example in which LQCD allows to decide between two very different classes of solutions of DSE's. And next we will see an example in which analytic meth-

\footnotetext{
${ }^{1} n_{f}+2$ if we count the strong $\mathrm{CP}$ term.
} 
ods provide the functional form of the ghost propagator in the deep infrared, thus allowing to extrapolate to zero momentum, where no direct LQCD calculation is possible.

\subsection{Notations and definitions}

In latin languages the translation of "ghost" starts with an "F", while "gluons" starts with a "G" in all languages we know. Therefore we use the following notations: the bare gluon propagator is written

$$
G_{\mu v}^{a b}\left(p^{2}, \Lambda^{2}\right) \equiv \frac{G\left(p^{2}, \Lambda^{2}\right)}{p^{2}} \delta_{a b}\left[\delta_{\mu v}-\frac{p_{\mu} p_{v}}{p^{2}}\right]
$$

where $G\left(p^{2}, \Lambda^{2}\right)$ is the bare gluon dressing function, $\Lambda$ is the ultraviolet cut-off, inverse lattice spacing $a^{-1}$ in the lattice case. The bare ghost propagator is written

$$
F^{a b}\left(p^{2}, \Lambda^{2}\right) \equiv \frac{F\left(p^{2}, \Lambda^{2}\right)}{p^{2}} \delta_{a b}
$$

where $F\left(p^{2}, \Lambda^{2}\right)$ is the bare ghost dressing function.

The corresponding renormalized quantities are labelled by the $R$ subscript:

$$
G_{R}\left(p^{2}, \mu^{2}\right) \equiv \lim _{\Lambda \rightarrow \infty} Z_{3}^{-1}\left(\mu^{2}, \Lambda\right) G\left(p^{2}, \Lambda^{2}\right) \quad F_{R}\left(p^{2}, \mu^{2}\right) \equiv \lim _{\Lambda \rightarrow \infty} \widetilde{Z}_{3}^{-1}\left(\mu^{2}, \Lambda\right) F\left(p^{2}, \Lambda^{2}\right)
$$

where $\mu$ is the renormalisation scale.

An important remark for the following is that $[2,3]$

$$
\begin{aligned}
& F(0, \Lambda)=\widetilde{Z}_{3}\left(\mu^{2}, \Lambda\right)\left(F_{R}\left(0, \mu^{2}\right)+\mathscr{O}\left(\frac{1}{\Lambda^{2}}\right)\right) \\
& G(0, \Lambda)=Z_{3}\left(\mu^{2}, \Lambda\right)\left(G_{R}\left(0, \mu^{2}\right)+\mathscr{O}\left(\frac{1}{\Lambda^{2}}\right)\right)
\end{aligned}
$$

In the MOM renormalisation scheme, the renormalised quantities are set equal to their tree value when the momentum is equal to the renormalisation scale:

$$
G_{R}\left(\mu^{2}, \mu^{2}\right)=F_{R}\left(\mu^{2}, \mu^{2}\right) \equiv 1
$$

whence, using eq. (1.3)

$$
Z_{3}\left(\mu^{2}, \Lambda^{2}\right)=G\left(\mu^{2}, \Lambda^{2}\right), \quad \widetilde{Z}_{3}\left(\mu^{2}, \Lambda^{2}\right)=F\left(\mu^{2}, \Lambda^{2}\right) .
$$

The bare ghost-ghost-gluon vertex is parametrised by

$$
\widetilde{\Gamma}_{v}^{a b c}(-q, k ; q-k)=i g_{0} f^{a b c}\left(q_{v} H_{1}(q, k)+(q-k)_{v} H_{2}(q, k)\right),
$$

where $k(q)$ is the incoming (outgoing) ghost momentum. Taylor's theorem [4] implies that the ghost-ghost-gluon vertex becomes trivial when the incoming momentum vanishes

$$
H_{1}(q, 0)+H_{2}(q, 0)=1
$$


This implies that if we take this kinematics to renormalise the ghost-ghost-gluon vertex, the vertex renormalisation constant is $\widetilde{z}_{1}=1$.

Exploiting this property we can define very simply "Taylor's coupling constant" by [5]

$$
\alpha_{T}\left(p^{2}, \Lambda^{2}\right)=\frac{g_{0}^{2}\left(\Lambda^{2}\right)}{4 \pi} F^{2}\left(p^{2}, \Lambda^{2}\right) G\left(p^{2}, \Lambda^{2}\right) .
$$

where $g_{0}^{2}\left(\Lambda^{2}\right)$ is the bare coupling constant. Notice that, while $g_{0}, F$ and $G$ depend logarithmically on the cut-off $\Lambda, \alpha_{T}$ only depends on it via inverse powers $O\left(1 / \Lambda^{2}\right)$.

Finally we assume, as everybody does, some simple power law in the deep infrared:

$$
G\left(p^{2}, \Lambda^{2}\right) \underset{p^{2} \rightarrow 0}{\propto}\left(p^{2}\right)^{\alpha_{G}} \quad F\left(p^{2}, \Lambda^{2}\right) \underset{p^{2} \rightarrow 0}{\propto}\left(p^{2}\right)^{\alpha_{F}} \Rightarrow \alpha_{T}\left(p^{2}, \Lambda^{2}\right) \underset{p^{2} \rightarrow 0}{\propto}\left(p^{2}\right)^{2 \alpha_{F}+\alpha_{G}}
$$

\section{Two classes of solutions to the ghost propagator Dyson-Scwinger equation}

Let us consider the ghost propagator Dyson-Schwinger equation (GPDSE). It was claimed by many authors trying to solve the DSE's that a general conclusion was that $2 \alpha_{F}+\alpha_{G}=0$ or, in other words that $\alpha_{T}\left(p^{2}\right) \rightarrow$ ct $>0$ when $p^{2} \rightarrow 0$. On the other hand many indications from lattice QCD show a strong vanishing of $\alpha_{T}\left(p^{2}\right)$, see a recent result at very small momenta in fig. 5 of [6].

Looking into details of the GPDSE we found that there were indeed two classes of solutions [7]

- Solution I: $2 \alpha_{F}+\alpha_{G}=0, \alpha_{T}\left(p^{2}\right) \underset{p^{2} \rightarrow 0}{\longrightarrow}$ ct $>0$ and $\alpha_{F}<0, F\left(p^{2}, \Lambda^{2}\right) \underset{p^{2} \rightarrow 0}{\longrightarrow} \infty$

- Solution II: $\alpha_{F}=0, F\left(p^{2}, \Lambda^{2}\right) \underset{p^{2} \rightarrow 0}{\longrightarrow}$ ct $>0$ and, using the lattice evidence that $\alpha_{G}>0,2 \alpha_{F}+$ $\alpha_{G}>0, \alpha_{T}\left(p^{2}\right) \rightarrow 0$

This is valid at fixed cut-off $\Lambda$. Similar conclusions hold for the renormalised quantities. Solution I is often called the "scaling solution" while solution II is called for some reason the "decoupling solution".

\subsection{Schematic proof of the existence of the two solutions}

The GPDSE writes in our notations as

$$
\frac{1}{F\left(k^{2}, \Lambda\right)}=1+g_{0}^{2} N_{c} \int \frac{d^{4} q}{(2 \pi)^{4}}\left(\frac{F\left(q^{2}, \Lambda\right) G\left((q-k, \Lambda)^{2}\right)}{q^{2}(q-k)^{4}}\left[\frac{(k \cdot q)^{2}}{k^{2}}-q^{2}\right] H_{1}(q, k, \Lambda)\right),
$$

Using Taylor's theorem eq. (1.8), completed with indications from perturbative QCD, we assume that in eq. (2.1) $H_{1}$ is regular: never vanishing nor infinite, and not too far from 1. In practice we will take it as a constant close to 1 . This hypothesis is rather usual. The r.h.s of eq. (2.1) is divergent at fixed $\Lambda$ since the integrand $G F$ decreases at large $q^{2}$ as $\alpha^{35 / 44}$ which is not enough to make the integral convergent. Therefore we prefer to regularize it by using a subtracted GPDSE:

$$
\begin{aligned}
\frac{1}{F\left(k^{2}\right)}-\frac{1}{F\left(k^{\prime 2}\right)} & =1+g_{0}^{2} N_{c} \int \frac{d^{4} q}{(2 \pi)^{4}}\left(\frac{F\left(q^{2}\right)}{q^{2}}\right)\left(\frac{G\left((q-k)^{2}\right)}{(q-k)^{4}}\left[\frac{(k \cdot q)^{2}}{k^{2}}-q^{2}\right]\right. \\
& \left.-\frac{G\left(\left(q-k^{\prime}\right)^{2}\right)}{\left(q-k^{\prime}\right)^{4}}\left[\frac{\left(k^{\prime} \cdot q\right)^{2}}{k^{\prime 2}}-q^{2}\right]\right) H_{1}
\end{aligned}
$$


where we have assumed $H_{1}$ to be a constant and omitted to write $\Lambda$. The r.h.s. now is convergent. Let us assume that we rescale all momenta by a common factor $\lambda \rightarrow 0$, we count the power behaviour of the 1.h.s and the r.h.s. From eq. (1.10) the 1.h.s behaves like $\left(\lambda^{2}\right)^{-\alpha_{F}}$ and the r.h.s as $\left(\lambda^{2}\right)^{\alpha_{G}+\alpha_{F}}$. Matching both sides leads to solution I: $2 \alpha_{F}+\alpha_{G}=0$. However, there is a loophole in this argument when $1 / F\left(p^{2}\right) \rightarrow 1 / F(0)>0$, i.e. when $\alpha_{F}=0$ since then the 1.h.s. vanishes to leading order. To get a relation we need to go to the subleading behaviour of $1 / F\left(p^{2}\right)$. We are then in the case of solution II: $\alpha_{F}=0$ and no constraint on $2 \alpha_{F}+\alpha_{G}$. This is the proof. More details can be found in [7].

\subsection{Numerical resolution of the GPDSE}

In order to understand better the relationship between these two classes of solutions we have performed a numerical solution of the GPDSE [8]. Since we consider only one DSE, we need additional inputs. Our inputs are:

- The gluon propagator is taken from lattice QCD. It is extrapolated to the large momenta using perturbative QCD formulae and to zero momentum assuming a finite, non zero limit, as strongly indicated by lattice QCD.

- The ghost-ghost gluon vertex is taken to be constant as justified above from Taylor's theorem.

- The coupling constant multiplies the vertex function which we assume to be constant. This product, a rescaled coupling constant, is taken as a free parameter.

We then fit this parameter to recover a solution in agreement with the ghost propagator computed by lattice QCD. This exercize can be performed, mutatis mutandis, with bare quantities or renormalized ones. In the latter case we define the rescaled coupling constant by

$$
\widetilde{g}^{2} \equiv N_{c} g_{R}^{2} \widetilde{z_{1}} H_{1 R}
$$

where $\widetilde{z_{1}}$ is the vertex renormalisation constant and our renormalisation scale is chosen to be 1.5 $\mathrm{GeV}$.

Our result is that there is one critical value of the rescaled coupling constant $\widetilde{g}_{c}^{2}=33.198$ for which the renormalised ghost dressing function diverges at zero momentum, solution I ("scaling"), while for all smaller $\widetilde{g}^{2}, F(0)$ is finite, solution II ("decoupling"). Fitting to the values of $F\left(k^{2}\right)$ from lattice data gives $\widetilde{g}^{2}=29$. The plots are shown in fig. 1 . Not surprisingly, the plot 2 shows that the product $F^{2} G$, proportional to Taylor's coupling constant, eq. (1.9), goes to a constant for the critical $\widetilde{g}_{c}^{2}$ and vanishes for any smaller $\widetilde{g}^{2}$, fitting lattice data for $\widetilde{g}^{2}=29$.

\subsection{Expansion of the ghost propagator at small momentum}

From the GPDSE one can derive the low momentum expansion of the Ghost dressing function in the case of solution II [8]. If we assume that the gluon propagator goes to a finite constant, which 


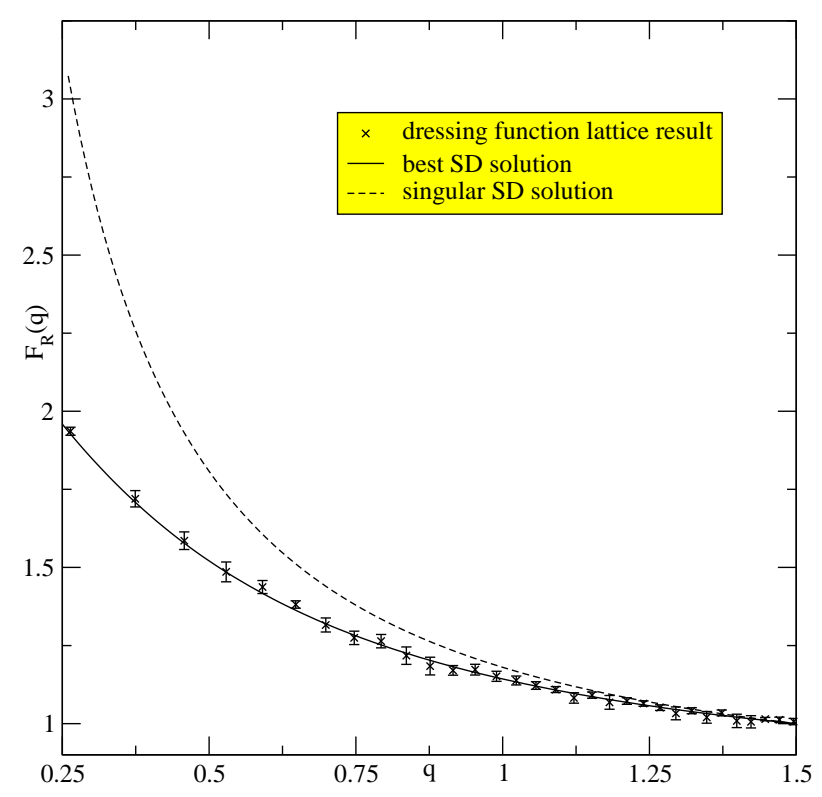

Figure 1: Comparison between the lattice $\mathrm{SU}(3)$ data at $\beta=5.8$ and with a volume $32^{4}$ for the ghost dressing function and our continuum SD prediction renormalised at $\mu=1.5 \mathrm{GeV}$ for $\widetilde{g}^{2}=29$. (solid line); the agreement is striking; also shown is the singular solution which exists only at $\widetilde{g}^{2}=33.198 \ldots$. (broken line), and which is obviously excluded.

implies that $\alpha_{T}\left(k^{2}\right) \propto k^{2}$, it takes a simple form:

$$
\begin{gathered}
F_{R}\left(k^{2}, \mu^{2}\right) \simeq F_{R}\left(0, \mu^{2}\right)\left(1+\frac{3 \widetilde{z}_{1} H_{1 R}}{16 \pi} \alpha_{T}\left(k^{2}\right) \log \left(k^{2}\right)\right) \\
F\left(k^{2}, \Lambda\right) \simeq F(0, \Lambda)\left(1+\frac{3 H_{1}}{16 \pi} \alpha_{T}\left(k^{2}\right) \log \left(k^{2}\right)\right)
\end{gathered}
$$

This formula, which can be refined [9], is very useful since it allows an extrapolation of lattice data down to zero momentum. This is an exemple in which an analytic method supports the numerical one.

\section{What do we learn from lattice QCD}

This will be a very brief section as everything has been covered in Teresa Mendes's talk. Let us just mention recent publications, which present results obtained with particularly large volumes and thus small momenta. What follows concerns bare Green functions at some finite cut-off. Cucchieri-Mendes have studied the $S U(2)$ case [10]: their fig. 2 shows a bending of the ghost dressing function perfectly compatible with solution II ("decoupling"). In [11] they consider the $\beta=0$ situation and exhibit bounds on the gluon propagator (their fig. 4). Bogolubsky et al. [6] consider $S U(3)$ : their fig. 2 shows that the gluon propagator goes to a non zero constant at zero momentum, fig. 4 shows also a bending of the ghost dressing function and fig. 5 clearly shows a vanishing of 


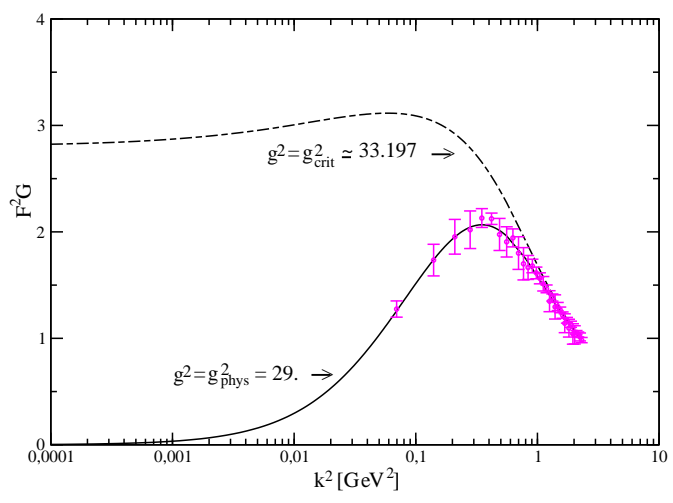

Figure 2: Comparison between our lattice $\mathrm{SU}(3)$ data at $\beta=5.8$ and with a volume $32^{4}$ for the product of gluon times ghost square dressing functions $G_{R}(k) F_{R}(k)^{2}$, renormalised at $\mu=1.5 \mathrm{GeV}$, and the corresponding curve for the continuum singular solution $\alpha_{G}+2 \alpha_{F}=0$, which exists only at $\widetilde{g}^{2} \simeq 33.198$, obviously excluded. Also shown is our continuum regular solution for $\widetilde{g}^{2}=29$ (solid line) for which the agreement is striking.

$\alpha_{T}$ at zero momentum. The general conlusion is that the gluon propagator goes to a non zero constant, the ghost dressing function may go to a finite non zero limit, and Taylor's coupling constant clearly vanishes at zero momentum. If the finiteness of the ghost dressing function is today only an indication, the vanishing of the coupling constant is compelling, thus contradicting solution I ("scaling"). Now, since the analytic GPDSE method says that there exists only these two classes of solutions, we may conclude that nature has chosen solution II and thus that the ghost dressing function must indeed go to a finite non zero constant at finite cut-off. As we see this is an exemple in which the LQCD numerical method allows to discriminate between two classes of solutions of the GPDSE.

One remark is in order here. We use the denomination "coupling constant" in a very general sense: any well defined quantity which in the ultraviolet is asymptotically equivalent to, say, $\alpha_{\overline{M S}}$, is eligible for the denomination "coupling constant". $\alpha_{T}$ defined in eq. (1.9) is obviously one of those [5]. Is this coupling constant convenient for phenomenological descriptions using tree level diagrams in the infrared? presumably no. If one aims at this phenomenology, as do the proponents of the "pinch technique" $[12,13]$, one could easily redefine a new eligible coupling constant by pulling a massive gluon propagator out for the gluon leg amputation of the ghost-ghost-gluon Green function used to build the coupling [14]. Thus $\alpha_{\text {new }}\left(k^{2}\right)=\alpha_{T}\left(k^{2}\right)\left(k^{2}+M^{2}\right) / k^{2}$ where $M$ could be the gluon mass ${ }^{2}$.

\section{Can the bare ghost dressing function be finite non zero ?}

This question was raised by Kondo's remark $[15,16]$ of a relation between the $k=0$ values of the ghost dressing function $F(k)$, Zwanziger's horizon function $h(k)$, Kugo's function $u(k)[17,18]$,

\footnotetext{
${ }^{2}$ We thank A.C. Aguilar, D. Binosi, J. Cornwall and J. Papavassiliou for this comment.
} 
and an additional function $w(k)$. Applying to this relation Zwanziger's horizon gap equation and assuming that $w(0)=0$ he derives the surprising result that $u(0)=-2 / 3$ and $\mathrm{F}(0)=3$, independently of the cut-off. The questions we will raise are: is this relation exact? does the prediction agree with lattice ? Is it compatible with renormalisability of QCD ? Our point of view is detailed in [19].

\subsection{Kondo's relations}

In this subsection we only consider bare quantities. One solution to the problem of Gribov's ambiguity,which was proposed by Zwanziger [20], consists in using the Gribov-Zwanziger partition function, which aims at restricting the Gribov copies [21] within the Gribov Horizon:

$$
Z_{\gamma}=\int[D A] \delta(\partial A) \operatorname{det}(M) e^{-S_{\mathrm{YM}}+\gamma \int d^{D} x h(x)},
$$

for the D-dimensional Euclidean Yang-Mills theory, where $S_{\mathrm{YM}}$ stands for the Yang-Mills action, $M$ is the Faddeev-Popov operator,

$$
M^{a b}=-\partial_{\mu} D_{\mu}^{a b}=-\partial_{\mu}\left(\partial_{\mu} \delta^{a b}+g f^{a b c} A_{\mu}^{c}\right)
$$

and $h(x)$ is Zwanziger's horizon function,

$$
h(x)=\int d^{D} y g f^{a b c} A_{\mu}^{b}(x)\left(M^{-1}\right)^{c e}(x, y) g f^{a f e} A_{\mu}^{f}(y) ;
$$

that restricts the integration over the gauge group to the first Gribov region, provided that the Gribov parameter, $\gamma$, is a positive number.

One defines then the function $u\left(k^{2}\right)$ which, at vanishing momentum, gives the Kugo-Ojima parameter, and the function $w\left(k^{2}\right)$ via the following identities.

$$
\begin{aligned}
\left\langle\left(D_{\mu}^{a b} c^{b}\right)\left(g f^{c d e} A_{v}^{d} \bar{c}^{e}\right)\right\rangle_{k} & =-\delta_{\mu v}^{T} \delta^{a c} u\left(k^{2}\right) ; \\
\left\langle c^{a}\left(g f^{d e f} A_{v}^{e} \bar{c}^{f}\right)\right\rangle_{k}^{1 \mathrm{PI}} & =i \delta^{a d} k_{v}\left(u\left(k^{2}\right)+w\left(k^{2}\right)\right) .
\end{aligned}
$$

From these definitions one obtains $[15,16]$ and [19] without any hypothesis about $u$ and $w$,

$$
\begin{aligned}
& u(0, \Lambda)=\frac{F(0, \Lambda)-1}{D-1}-\frac{D}{D-1}\left[\frac{\langle h(0)\rangle_{k=0}}{D\left(N^{2}-1\right)}\right] \\
& w(0, \Lambda)=-1-u(0, \Lambda)+\frac{1}{F(0, \Lambda)}=-\frac{F(0, \Lambda)+(D-2)}{D-1}+\frac{1}{F(0, \Lambda)}+\frac{D}{D-1}\left[\frac{\langle h(0)\rangle_{k=0}}{D\left(N^{2}-1\right)}\right]
\end{aligned}
$$

\subsection{No finite $F(0, \Lambda)$ is possible at large cut-off $\Lambda$}

If we use Zwanziger's gap equation:

$$
\langle h(x)\rangle_{\gamma}=\left(N^{2}-1\right) D .
$$

the functions $u(0)$ and $w(0)$ become, from eq. (4.5), a function of the bare $F(0)$ plotted in Fig. 3. The current lattice solutions for the bare ghost dressing functions at vanishing momentum lie inside the green dotted square. The apparent approximate agreement of lattice results with Kondo's solution is nevertheless misleading and due to the moderate cut-off value on the lattices. 


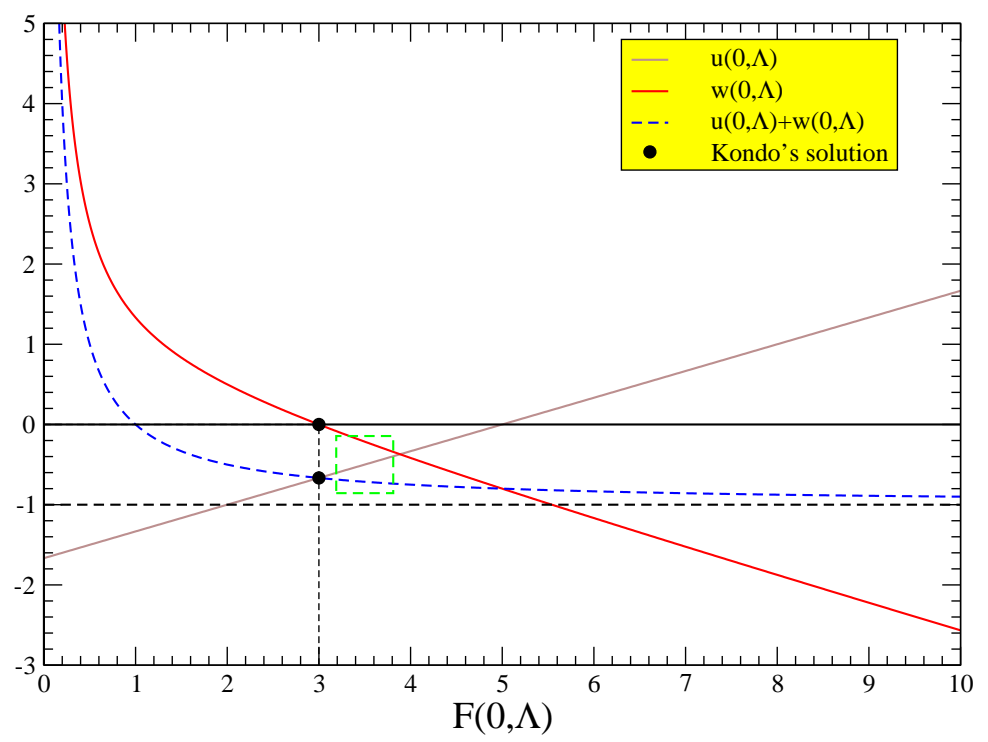

Figure 3: The solutions for $u(0, \Lambda)$ and $w(0, \Lambda)$ plotted as a function of $F(0, \Lambda)$ under the assumption that the horizon gap equation is valid.

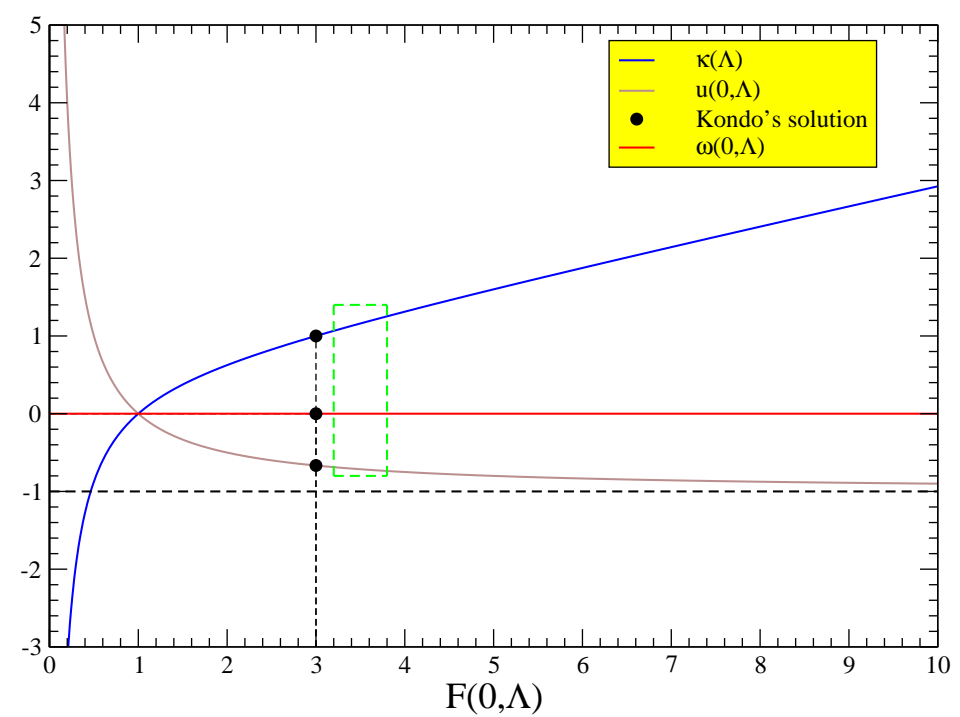

Figure 4: The same plot shown in fig. 3 but $w(0, \Lambda)$ is required to be zero and the gap equation is relaxed by a multiplicative factor $\kappa(\Lambda)$, as explained in the text. $\kappa(\Lambda)$ is plotted on the solid blue line. Again, current lattice estimates lie inside the green dotted square. 
Indeed let us assume a fixed renormalised $F_{R}\left(0, \mu^{2}\right)$. The plot in fig. 3 can then be understood, as a function of the $\widetilde{Z}_{3}\left(\mu^{2}, \Lambda\right)$ at fixed $\mu^{2}$ as soon as inverse powers of $\Lambda$ become negligible in front of logarithms, since, from eq. (1.3), eq. (1.4),

$$
\widetilde{Z}_{3}\left(\mu^{2}, \Lambda\right)=\frac{F(0, \Lambda)}{F_{R}\left(0, \mu^{2}\right)}+\mathscr{O}\left(\frac{1}{\Lambda^{2}}\right) .
$$

The large cut-off dependance of $\widetilde{Z}_{3}$ is known to be:

$$
\frac{\widetilde{Z}_{3}\left(\mu^{2}, \Lambda\right)}{\widetilde{Z}_{3}\left(\mu^{2}, \Lambda_{0}\right)}=\left(\frac{\log \left(\Lambda / \Lambda_{\mathrm{QCD}}\right)}{\log \left(\Lambda_{0} / \Lambda_{\mathrm{QCD}}\right)}\right)^{9 / 44}(1+\mathscr{O}(\alpha))
$$

$\widetilde{Z}_{3}\left(\mu^{2}, \Lambda\right) \rightarrow \infty$ when $\Lambda \rightarrow \infty$.

Then the infinite cut-off limit is the limit at infinity on the horizontal axis of fig. 3 . The particular solution proposed in ref. $[15,16]$ (black circles), obtained by imposing $w(0, \Lambda)=0$, corresponds to the intersection of $u+w$ and $u$. It cannot hold when $\widetilde{Z}_{3} \rightarrow \infty$. Notice that the hypothesis of a finite bare $F(0)$ with a vanishing $F_{R}(0)$ does not hold either since then $F(0, \Lambda)=$ $\widetilde{Z}_{3} F_{R}(0)+\mathscr{O}\left(1 / \Lambda^{2}\right)=\mathscr{O}\left(1 / \Lambda^{2}\right)$ and consequently $F(0, \Lambda)$ vanishes when $\Lambda \rightarrow \infty$.

Notice also from fig. 3 that a finite $w(0, \Lambda)$ is not possible at the large cut-off limit.

We should now take into account that gap equation (4.6) is a consequence of Gribov-Zwanziger modification of the Yang-Mills action eq. (4.1). This is not what is done in LQCD, although lattice gauge fixing also restricts the Gribov copies within Gribov's horizon. Therefore we believe that condition eq. (4.6) has no reason to be fulfilled in LQCD and maybe not at all in QCD. Let us define $\kappa(\Lambda)$ such that

$$
\langle h(0)\rangle_{k=0}=\lim _{k \rightarrow 0} \frac{1}{V_{D}} \int d^{D} x\langle h(x)\rangle e^{i k \cdot x}=\kappa(\Lambda)\left(N^{2}-1\right) D .
$$

If the gap equation eq. (4.6) is thus relaxed it becomes possible to keep $w(0)$ finite, a result derived in $[22,23]$ in the Landau background gauge. We show the solution when $w(0)=0$ on the fig. 4. Nothing changes concerning the fact that the infinite cut-off limit is at infinity on the horizontal axis. Our conclusion still remains valid: is not possible to have a finite $F(0, \Lambda)$ in the large $\Lambda$ limit

\section{Conclusion}

In fig. 5 we perform an extrapolation of the lattice bare ghost dressing function using the small momentum expansion [19] shortly explained in eq. (2.4) of section 2.3. The data for the two larger lattice volumes are taken from ref. [6] and the others from refs. [24, 8]. The fit with formula eq. (2.4) is rather good in its range of validity (small momentum). We also notice that the different lattice results seem to agree rather well although they correspond to different $\beta$ values i.e. different lattice spacings. One may feel happy and claim that we have a good scaling invariance. But this is a wrong statement from a misleading observation.

Indeed, remember that the lattice spacing is the inverse of the cut-off in lattice regularisation. From eq. (4.8) we know that when the lattice spacing goes to zero, $(\Lambda \rightarrow \infty), F(0, \Lambda) \propto \beta^{9 / 44}$. On the whole range of lattice spacings considered in fig. 5 , although the cut-off varies by more that a 


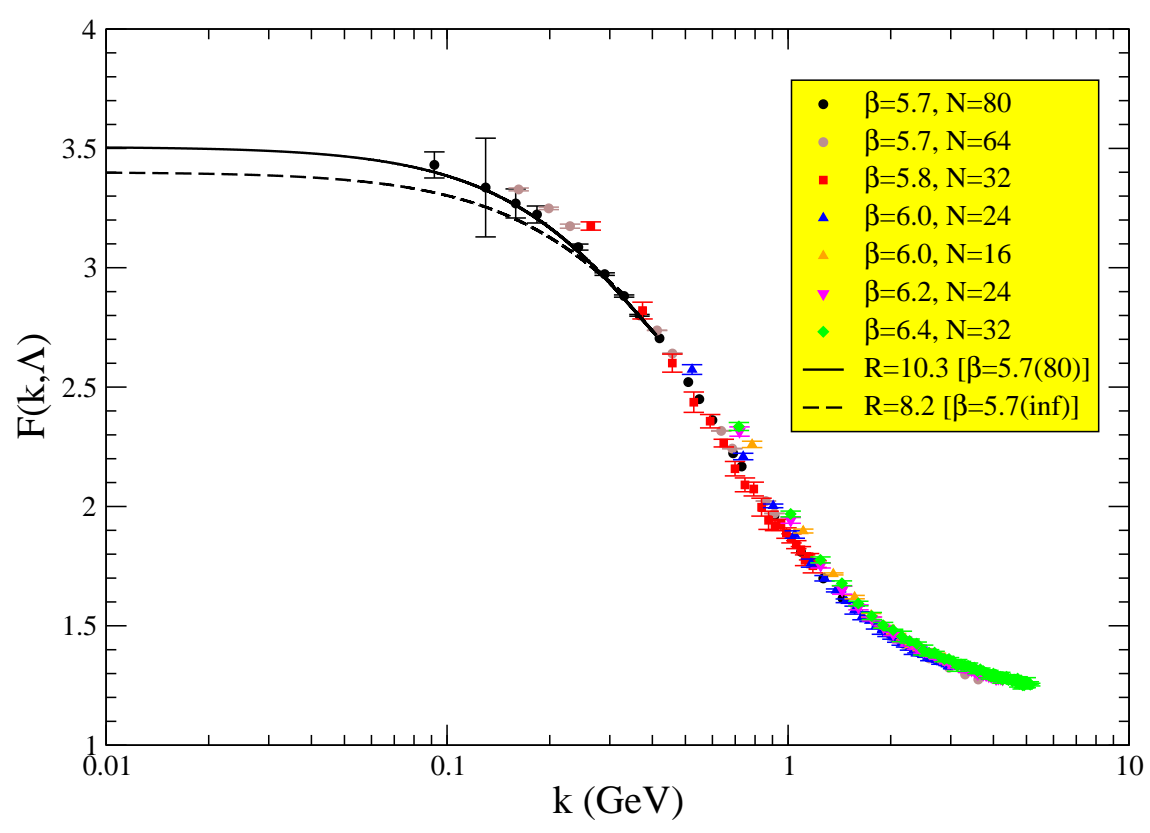

Figure 5: Bare ghost dressing function estimated from different lattice data sets. The solid line is for the best fit with the small-momentum expansion with $R\left(\beta=5.7\left(80^{4}\right)\right)$ and the dashed one stands for the best fit with $R(\beta=5.7, \infty)$.

factor of $3, \beta^{9 / 44}$ varies only by $2.5 \%$. This is why this variation is obscured by statistical errors ${ }^{3}$ in fig. 5. This fake "scaling invariance" hides the truth: $F\left(k^{2}, \Lambda\right)$ rises very slowly to infinity when $\Lambda \rightarrow \infty$ i.e. $\beta \rightarrow \infty$.

Bare values depend dramatically, although slowly, on the cut-off and have no real meaning unless the cut-off is specified. What makes really sense and has well defined limits at infinite cut-off (vanishing lattice spacing) are renormalised quantities [2, 3]. If we choose $1.5 \mathrm{GeV}$ as the renormalisation scale, we get from lattice the gross estimate

$$
F(1.5 \mathrm{GeV}) \equiv \widetilde{Z_{3}} \simeq 1.6 \text { whence } F_{R}(0,1.5 \mathrm{GeV}) \simeq 2.2
$$

Altogether, combining all which has been discussed here, our conclusion concerning the ghost dressing function is

- The renormalised ghost dressing function $F_{R}\left(0, \mu^{2}\right)$ has a finite limit at vanishing momentum, $F_{R}\left(0,(1.5 \mathrm{GeV})^{2}\right) \simeq 2.2$. It is a positive decreasing function at small momenta, probably also decreasing for all momenta.

- The bare ghost dressing function $F\left(k^{2}, \Lambda\right)$ goes very slowly to infinity at infinite $\Lambda$ for all momenta.

\footnotetext{
${ }^{3}$ Indeed there is a trend of the largest cut-off, $\beta=6.4$, to lie above the others, but this is hardly visible.
} 


\section{References}

[1] V. N. Gribov, Eur. Phys. J. C 10 (1999) 91 [arXiv:hep-ph/9902279].

[2] T. Reisz, Nucl. Phys.B318 (1989) 417

[3] M. Lüscher, arXiv:hep-lat/9802029.

[4] J. C. Taylor, Nucl. Phys. B 33 (1971) 436.

[5] L. von Smekal, K. Maltman and A. Sternbeck, arXiv:0903.1696 [hep-ph].

[6] I. L. Bogolubsky, E. M. Ilgenfritz, M. Müller-Preussker and A. Sternbeck, Phys. Lett. B 676 (2009) 69 [arXiv:0901.0736 [hep-lat]].

[7] Ph. Boucaud, J. P. Leroy, A. Le Yaouanc, J. Micheli, O. Pène and J. Rodríguez-Quintero, JHEP 0806 (2008) 099 [arXiv:0803.2161 [hep-ph]].

[8] Ph. Boucaud, J. P. Leroy, A. L. Yaouanc, J. Micheli, O. Pène and J. Rodríguez-Quintero, JHEP 0806 (2008) 012 [arXiv:0801.2721 [hep-ph]].

[9] Ph. Boucaud et al. "The low momentum ghost dressing function and the gluon mass" in preparation.

[10] A. Cucchieri and T. Mendes, Phys. Rev. D 78 (2008) 094503 [arXiv:0804.2371 [hep-lat]].

[11] A. Cucchieri and T. Mendes, arXiv:0904.4033 [hep-lat].

[12] D. Binosi and J. Papavassiliou, Phys. Rept. 479 (2009) 1 [arXiv:0909.2536 [hep-ph]].

[13] J. M. Cornwall, arXiv:0904.3758 [hep-ph].

[14] A. C. Aguilar et al., Phys. Rev. D 80 (2009) 085018 [arXiv:0906.2633 [hep-ph]].

[15] K. I. Kondo, Phys. Lett. B 678 (2009) 322 [arXiv:0904.4897 [hep-th]].

[16] K. I. Kondo, arXiv:0907.3249 [hep-th].

[17] T. Kugo and I. Ojima, Prog. Theor. Phys. Suppl. 66 (1979) 1.

[18] T. Kugo, arXiv:hep-th/9511033.

[19] Ph. Boucaud, J. P. Leroy, A. L. Yaouanc, J. Micheli, O. Pène and J. Rodríguez-Quintero, arXiv:0909.2615 [hep-ph].

[20] D. Zwanziger, Nucl. Phys. B 323 (1989) 513.

[21] V. N. Gribov, Nucl. Phys. B 139 (1978) 1.

[22] A. C. Aguilar, D. Binosi and J. Papavassiliou, arXiv:0907.0153 [hep-ph].

[23] P. A. Grassi, T. Hurth and A. Quadri, Phys. Rev. D 70 (2004) 105014 [arXiv:hep-th/0405104].

[24] Ph. Boucaud et al., Phys. Rev. D 72 (2005) 114503 [arXiv:hep-lat/0506031]; 